\title{
Efficiency and safety of one-year anti-TNF- $\alpha$ treatment in Crohn's disease: a Polish single-centre experience
}

\author{
Marcin Sochal ${ }^{1,2}$, Monika Krzywdzińska ${ }^{1}$, Agata Gabryelska ${ }^{1,2}$, Renata Talar-Wojnarowska ${ }^{1}$, Ewa Małecka-Panas ${ }^{1}$ \\ ${ }^{1}$ Department of Digestive Tract Diseases, Medical University of Lodz, Lodz, Poland \\ ${ }^{2}$ Department of Sleep Medicine and Metabolic Disorders, Medical University of Lodz, Lodz, Poland
}

Gastroenterology Rev 2020; 15 (2): 156-160

DOI: https://doi.org/10.5114/pg.2019.90079

Key words: Crohn's disease, infliximab, adalimumab, effectiveness of biological treatment, side effects.

Address for correspondence: Marcin Sochal MD, Department of Sleep Medicine and Metabolic Disorders, Medical University of Lodz, 6/8 Mazowiecka St, 92-215 Lodz, Poland, phone: +48 42678 18 00, e-mail: sochalmar@gmail.com

\begin{abstract}
Introduction: Anti-TNF- $\alpha$ therapy of Crohn's disease (CD) represents considerable progress in inflammatory bowel disease (IBD) treatment; however, many patients still require surgical intervention. The Polish National Insurance Fund currently only covers up to 2 years of infliximab (IFX) therapy in CD patients and 1 year of adalimumab (ADA).

Aim: To estimate the effectiveness and side effects of the anti-TNF- $\alpha$ Polish therapeutic program in CD patients.

Material and methods: In this retrospective study, medical documentation of 80 CD patients treated with anti-TNF- $\alpha$ (IFX or ADA) was analysed. Fifty-two patients finished 1 year of therapy, and 28 individuals did not complete it due to lack of response to treatment or severe side effects.

Results: After treatment, 27 (67.50\%) patients achieved a semi-annual remission and 14 (35\%) achieved yearly remission. Twenty percent of patients experienced severe side effects such as anaphylactic shock, pneumonia, shingles, or upper respiratory tract infections. A strong negative correlation between the number of patients in remission and the period since therapy termination $(r=-0.996, p<0.001)$ was found. During the 1-year follow-up, 20 patients were re-enrolled in the biological therapy program (the median time to next therapy was 231 days IQR: 126.5-300.5)

Conclusions: Anti-TNF- $\alpha$ treatment in CD is relatively safe. The restricted time period of the therapy affects the clinical course of the disease and entails the need to resume biological therapy.
\end{abstract}

\section{Introduction}

Crohn's disease (CD) is a chronic autoimmune disease of the digestive tract, with poorly understood aetiopathogenesis. In the last 20 years there has been a significant increase in the incidence of inflammatory bowel disease (IBD) [1, 2]. During exacerbation, mainly steroids are used, which do not allow achievement of long-term remission, often causing dependence and significant side effects (SE) [3].

The biological therapy of CD patients is mainly based on tumour necrosis factor- $\alpha$ monoclonal antibodies (anti-TNF- $\alpha$ ). In Poland, available preparations include infliximab (IFX), which is a human-mouse antibody and adalimumab (ADA), a recombinant antibody
$[4,5]$. Biological treatment represents a significant advance in the treatment of CD patients. These drugs reduce the number of surgical interventions and lead to endoscopic remission of the disease [6-8].

The guidelines do not regulate when and in which patients anti-TNF- $\alpha$ therapy might be stopped, due to insufficient literature and divergent results $[9,10]$. The Polish National Insurance Office currently funds IFX therapy in CD patients for up to 2 years and ADA up to 1 year.

\section{Aim}

The aim of the study was to estimate the effectiveness and SE of the anti-TNF- $\alpha$ Polish therapeutic program in CD patients. 


\section{Material and methods}

In this retrospective study the medical documentation was analysed of CD patients treated at the Department of Digestive Tract Diseases, Medical University of Lodz, Poland in the period from May 2008 to August 2017. In these patients, anti-TNF- $\alpha$ therapy (IFX or ADA) was administered for 1 year, in accordance with the characteristics of the medical product. The dose of the drug was not increased during the therapy period.

Enrolment criteria to biological therapy were: severe course of the disease (disease activity expressed according to Crohn's Disease Activity Index (CDAI) $>300$ ), the presence of fistulas, ineffectiveness, intolerance, or contraindications to immunomodulatory drugs and steroids.

Data were collected on the duration of therapy, $\mathrm{SE}$, concomitant drugs, and the course of the disease: previous operations, complications of the disease (fistulas, arthritis, erythema nodosum) and the disease duration. Six and 12 months after therapy termination, the disease activity was assessed based on the information contained in the medical documentation: surgical interventions, steroids, immunomodulatory drugs, and biological therapies. The study received a positive opinion from the local Bioethics Commission (RNN/372/18/KE).

\section{Statistical analysis}

The statistical analysis was performed with Statistica 13.1 software. The Mann-Whitney $U$ test was used for quantitative variables, and the $\chi^{2}$ test was used for nominal variables. Correlations between variables were examined using the Pearson test. Additionally, the Kaplan-Meier estimator was used in the analysis. The level of statistical significance was $p<0.05$.

\section{Results}

The medical histories of 80 CD patients (37 women and 43 men) treated with anti-TNF- $\alpha$ in the Department of Digestive Tract Diseases were analysed. The median age at the start of therapy was 31.5 (IQR: 24-40) years (Figure 1). Forty-two patients (52.50\%) received IFX and 38 (47.50\%) recieved ADA. In 11 (13.75\%) patients only induction therapy was administered due to the lack of response to the treatment and severe respiratory infection (one case).

Maintenance therapy was not completed by 17 patients (seven treated with ADA and 10 with IFX). The most common cause of premature termination of therapy was disease exacerbation ( 6 cases, therein: two surgical intervention and 2 patients with abscess formation, in 2 patients the method of pharmacological treatment was changed). In addition, severe respiratory infections (5 cases), in 1 patient -- Clostridium difficile infection and in 1 patient - pregnancy. In four patients, data about the reason for early termination of therapy was unavailable.

Sixteen (20\%) patients had experienced SE such as infections of the respiratory tract $(62.50 \%)$, shingles, or mononucleosis (one case of each). SE occurred with a similar frequency in patients treated with ADA and with IFX ( $8 / 38$ vs. $8 / 42, p=0.823)$. Seven patients (8.75\% of all patients) stopped therapy due to SE.

The remission time of $52 \mathrm{CD}$ patients who underwent induction therapy and 1-year maintenance was analysed. Demographic data and clinical characteristics of patients are presented in Table I. Thirty (57.69\%) patients used immunomodulatory drugs in addition to biological therapy. History of surgical operations before biological therapy concerned 16 (30.77\%) patients. There was no significant difference between patients treated with IFX and ADA regarding age at start of therapy, age at diagnosis, gender, or additional use of immunomodulators.

Immediately after 1 -year maintenance therapy, $47(90.38 \%)$ patients were in remission, after 6 months - 38 (73.08\%), and after 12 months - 21 (40.38\%) (Figure 1). A strong negative relationship between the number of patients in remission of the disease and the time (months) from the end of therapy $(r=-0.996$, $p<0.001$ ) was found (Figure 2 A). After 9 months, only half of the patients maintained in remission as shown by the Kaplan-Meier estimator (Figure 2 B). Twenty (38.46\%) patients were re-enrolled in the biological therapy program for 1 year, representing $64.52 \%$ of patients, who did not maintain annual remission following

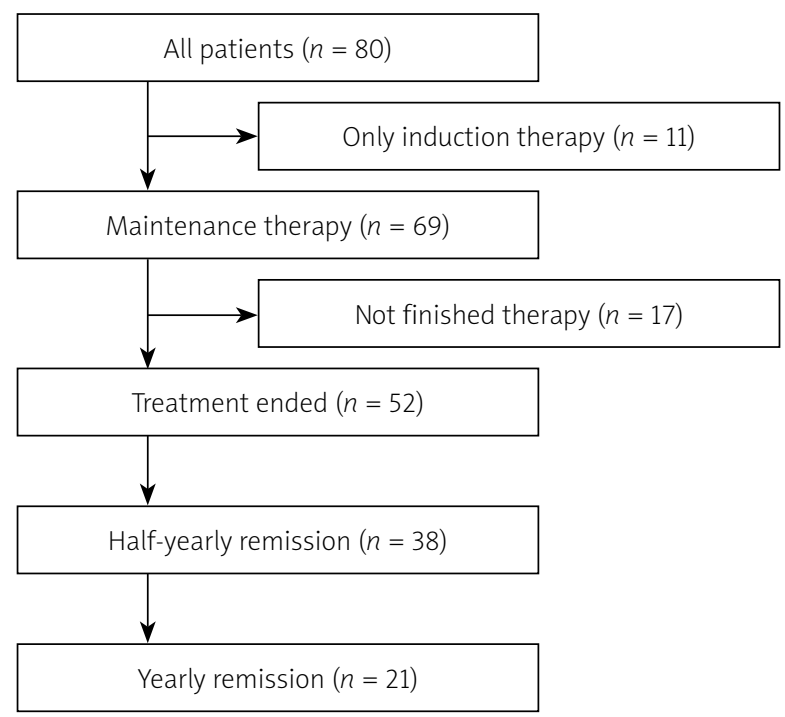

Figure 1. Flowchart of the study 
Table I. Baseline characteristics of patients included in the analysis along with the division into patients receiving ADA or IFX

\begin{tabular}{|c|c|c|c|c|}
\hline Parameter & All & ADA & IFX & $P$-value \\
\hline$N$ & 52 & 29 & 23 & - \\
\hline Age, median (IQR) & $33(26-42.5)$ & $33(25-54)$ & $32(26-39)$ & 0.912 \\
\hline Age at diagnosis, median (IQR) & $27(20-39.5)$ & $21(18-26)$ & $40(29-45)$ & 0.555 \\
\hline Women, $n(\%)$ & $23(44.23)$ & $14(48.28)$ & $9(39.13)$ & 0.51 \\
\hline Other chronic diseases, $n(\%)$ & $19(36.54)$ & $12(41.38)$ & $7(30.43)$ & 0.416 \\
\hline Previous surgical procedures, $n(\%)$ & $16(30.77)$ & $9(31.03)$ & $7(30.43)$ & 0.963 \\
\hline Use of immunomodulators, $n(\%)$ & $30(57.69)$ & $19(65.52)$ & $11(47.83)$ & 0.2 \\
\hline
\end{tabular}

$A D A$ - adalimumab, IFX - infliximab, IQR - interquartile range, $n$ - number of participants.

A

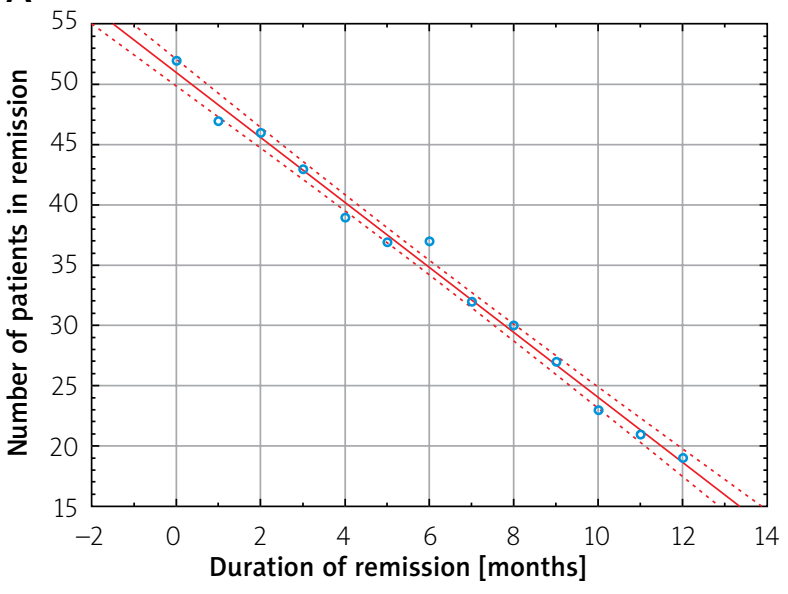

B

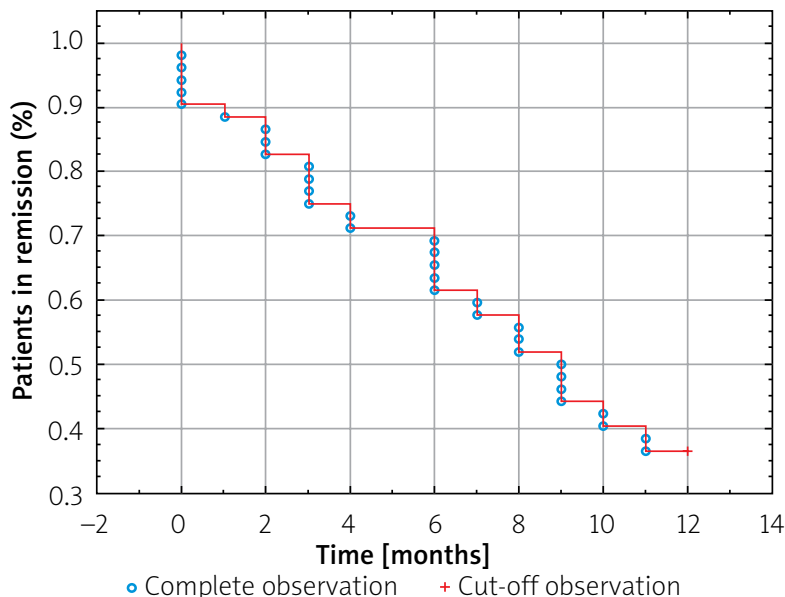

Figure 2. A - Negative correlation between the time from the end of therapy and the number of patients in remission $(r=-0.9958, p<0.001)$. B - Kaplan-Meier curves for remission of Crohn's disease

the end of therapy (the median time to next therapy: 231 days IQR:126.5-300.5).

Fifty-two (65\%) patients completed the entire planned treatment period without serious SE, additional hospitalisations, and surgical procedures.

\section{Discussion}

Anti-TNF- $\alpha$ therapy effectively introduces patients to remission; however, after the end of treatment, the condition of a large number of patients worsens. It has been shown that the annual risk of exacerbation from discontinuing the use of biological therapy is $59.62 \%$ (95\% Cl: 41.52-61.44); the number of patients in remission decreased in a linear manner. In the meta-analysis by Kennedy et al. this risk was estimated at $36 \%$ (95\% Cl: 29-44\%) [11]. Waugh et al. observed the incidence of disease relapse for 7 years after stopping treatment with IFX. Half of the patients suffered from the disease exacerbation after 16 months [12]. In our study the same rate was achieved after only 9 months, and patients were treated for the same period of time. Another study has shown that half of the CD patients were in remission 17 months after the end of therapy [13]. The differences could be caused by another definition of remission (clinical/endoscopic, with or without the use of immunomodulators). The duration of therapy was also different - in one study it lasted from 1 to 67 months [12]. In another meta-analysis, in which patients with CD were treated with IFX for at least 1 year, the annual risk of recurrence was similar to that shown in our study (43.9 $\pm 5.0 \%)$ [14].

The number of patients in remission decreased during the year after the end of therapy by $24.62 \%$ in a linear manner. In other studies, in which maintenance therapy was continued for 1 year, the number of patients in remission decreased by only $6-9 \%[15,16]$. This indicates the need for longer maintenance therapy, at least in selected patients whose prognosis is worse. 
We showed that up to $38.46 \%$ of patients within 1 year of the end of therapy were again included in anti-TNF- $\alpha$ induction therapy. It should be examined whether this approach affects the quality of life of patients and SE. An important aspect in biological re-treatment is the concentration of anti-TNF- $\alpha$ antibodies that may be responsible for poor reaction to treatment and hypersensitivity. A decrease in IFX concentration is associated with a high level of these antibodies [17]. However, the research is contradictory regarding whether its level lasts for many years or falls to an undetectable level $[18,19]$. Studies have shown that up to $90 \%$ of patients respond well to re-treatment. This may be related to the satisfactory response of these patients to the previous therapy and the lack of hypersensitivity to these drugs [17].

From 2017, the Polish biological treatment program for CD patients allows the duration of IFX therapy to be up to 24 months. It will be interesting to see whether the extended treatment time affects long-term remission.

Approximately $9 \%$ of patients stopped therapy due to SE. Similarly, in another study IFX treatment was stopped in $11 \%$ of respondents due to SE [18]. Lehtola et al. showed that $21 \%$ of respondents had SE during 2 years of treatment and stopped the therapy for this reason [19]. The small differences are probably due to the longer duration of treatment in the described studies.

Anti-TNF- $\alpha$ therapy for patients with CD is relatively safe and effective. After 1 year of biological therapy exacerbations follow, and resuming the treatment is often necessary. A restricted time period of the therapy may contribute to further exacerbate the disease and entails the need to reassume biological therapy. Longer maintenance biological therapy in CD should be considered.

\section{Acknowledgments}

Supported by a grant from the Medical University of Lodz (\#564/1-000-00/564-20-024 to MS) and a grant from the National Science Centre (\#2018/31/N/ NZ5/03715 to MS).

\section{Conflict of interest}

The authors declare no conflict of interest.

\section{References}

1. van den Heuvel TRA, Jeuring SFG, Zeegers MP, et al. A 20-year temporal change analysis in incidence, presenting phenotype and mortality, in the Dutch IBDSL Cohort-Can diagnostic factors explain the increase in IBD incidence? J Crohn's Colitis 2017; 11: 1169-79.

2. Molodecky NA, Soon IS, Rabi DM, et al. Increasing incidence and prevalence of the inflammatory bowel diseases with time, based on systematic review. Gastroenterology 2012; 142: 4654.e42.

3. Yanai $\mathrm{H}$, Hanauer SB. Assessing response and loss of response to biological therapies in IBD. Am J Gastroenterol 2011; 106: 685-98.

4. Golik M, Kurek M, Poteralska A, et al. Working group guidelines on the nursing roles in caring for patients with Crohn's disease and ulcerative colitis in Poland. Gastroenterol Rev 2014; 9: 179-93.

5. Bosani M, Ardizzone S, Porro GB. Biologic targeting in the treatment of inflammatory bowel diseases. Biologics 2009; 3: 77-97.

6. Domżał-Magrowska D, Talar-Wojnarowska R, Kotynia J, et al. Efficacy and safety of biological treatment in Crohn's disease: our experience. Gastroenterol Rev 2011; 6: 304-9.

7. Costa J, Magro F, Caldeira D, et al. Infliximab reduces hospitalizations and surgery interventions in patients with inflammatory bowel disease: a systematic review and meta-analysis. Inflamm Bowel Dis 2013; 19: 98-110.

8. Lopez A, Ford AC, Colombel JF, et al. Efficacy of tumour necrosis factor antagonists on remission, colectomy and hospitalisations in ulcerative colitis: meta-analysis of placebo-controlled trials. Dig Liver Dis 2015; 47: 356-64.

9. Gomollón F, Dignass A, Annese V, et al. $3^{\text {rd }}$ European Evidence-based Consensus on the Diagnosis and Management of Crohn's Disease 2016: Part 1: Diagnosis and Medical Management. J Chron's Colitis 2017; 11: 3-25.

10. Łodyga M, Eder P, Bartnik W, et al. Guidelines for the management of Crohn's disease. Recommendations of the Working Group of the Polish National Consultant in Gastroenterology and the Polish Society of Gastroenterology. Gastroenterol Rev 2012; 7: 317-38.

11. Kennedy NA, Warner B, Johnston EL, et al. Relapse after withdrawal from anti-TNF therapy for inflammatory bowel disease: an observational study, plus systematic review and meta-analysis. Aliment Pharmacol Ther 2016; 43: 910-23.

12. Waugh AWG, Garg S, Matic K, et al. Maintenance of clinical benefit in Crohn's disease patients after discontinuation of infliximab: long-term follow-up of a single centre cohort. Aliment Pharmacol Ther 2010; 32: 1129-34.

13. Kaymak T, Moriconi M, Niess JH, et al. Low discontinuation rate of infliximab treatment in steroid-dependent/refractory Crohn's disease patients. Inflamm Intest Dis 2018; 2: 171-9.

14. Louis E, Mary J, Vernier-Massouille G, et al. Maintenance of remission among patients with Crohn's disease on antimetabolite therapy after infliximab therapy is stopped. Gastroenterology 2012; 142: 63-70.

15. Caviglia R, Ribolsi M, Rizzi M, et al. Maintenance of remission with infliximab in inflammatory bowel disease: efficacy and safety long-term follow-up. World J Gastroenterol 2007; 13: 38-44.

16. Sandborn WJ, Hanauer SB, Rutgeerts PJ, et al. Adalimumab for maintenance treatment of Crohn's disease: results of the CLASSIC II trial. Gut 2007; 56: 1232-9.

17. Ben-Horin S, Mao R, Qiu Y, et al. Discontinuation of biological treatments in inflammatory bowel disease: a concise pragmatic review. J Clin Gastroenterol 2018; 52: 6-12. 
18. Hamzaoglu H, Cooper J, Alsahli M, et al. Safety of infliximab in Crohn's disease: a large single-center experience. Inflamm Bowel Dis 2010; 16: 2109-16.

19. Lehtola E, Haapamäki J, Färkkilä MA. Outcome of inflammatory bowel disease patients treated with TNF-alpha inhibitors: two-year follow-up. Scand J Gastroenterol 2016; 51: 1476-81.

Received: 15.07 .2019

Accepted: 8.08.2019 